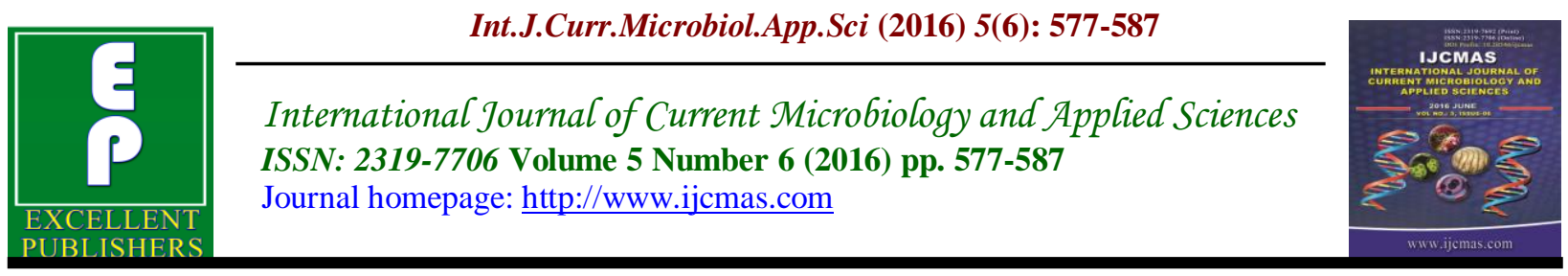

Original Research Article

http://dx.doi.org/10.20546/ijcmas.2016.506.062

\title{
Bacterial Etiology: Significant In Allergic Respiratory Diseases
}

\author{
Kishori S. Tarfe ${ }^{1 *}$ and Sunita D. Deshpande ${ }^{2}$ \\ ${ }^{1}$ Department of Biotechnology, Smt CHM College, Ulhasnagar, Dist Thane, Maharashtra, India \\ ${ }^{2}$ Former Professor, T. N. Medical College \& B. Y. L. Nair charitable hospital, Mumbai, \\ Maharashtra, India \\ *Corresponding author
}

\section{A B S T R A C T}

\section{Keywords}

Asthma,

Allergen,

Airway hyperresponsiveness, inflammation.

Article Info

Accepted:

20 May 2016

Available Online:

10 June 2016
Amongst allergic respiratory diseases allergic rhinitis \& asthma are the most common. Asthma is a chronic inflammatory disease of the airways which in susceptible individuals causes recurrent episodes of wheezing, breathlessness, chest tightness \& cough. Inflammation can cause increased airway hyperresponsiveness which can lead to infections by bacteria including atypical bacteria, fungi \& viruses. Microbial infections associated with allergic respiratory infections increase severity \& duration of the disease as well as they themselves act as an allergen. Therefore their treatment with appropriate antimicrobials is essential. Total 300 patients suffering from Asthma which is most common allergic respiratory disease were investigated for detection of bacterial pathogens in their sputum samples. Bacterial pathogens were isolated in $20 \%$ cases, which were M. catarrhalis, $S$. aureus, S. pyogenes, K. pneumoniae, \& P. aeruginosa. $M$. catarrhalis was the most common isolate found in $19 \%$ cases followed by $S$. aureus in $28.33 \%$ cases, K.pneumoniae \& P. aeruginosa in $16.66 \%$ cases each \& S. pyogenes in $6.6 \%$ cases. Antibiotic sensitivity testing was performed to determine their sensitivity pattern \& it was observed that $M$. catarrhalis \& S. pyogenes were sensitive to all the antibiotics tested. Other isolates showed variable susceptibility pattern. These isolates showed susceptibility to Aminoglycosides \& cephalosporin group. Various bacteria play a major role in severity of exacerbations, symptoms \& overall progress of allergic respiratory disease such as asthma. These bacterial infections can be diagnosed \& treated with appropriate \& specific antibiotics. This antibiotic treatment will help in fast recovery of patients with reduced duration of corticosteroid consumption of patients.

\section{Introduction}

The respiratory system is the most common site for infection by pathogenic microorganisms. Though these infections are common and usually mild, they are frequently taken for granted; they represent an immense disease burden on our society. The respiratory tract becomes infected frequently as it is in direct contact with the environment and is continuously exposed to microorganisms suspended in the air we breathe. Some of these microorganisms are highly virulent and only a 
few of them are needed to infect even healthy person. However, most of these microorganisms do not cause infection unless other factors interfere with the defenses. The warm, moist environment of the respiratory tract is an ideal place for growth of microorganisms' (Schaechter et al., 1989).

Amongst allergic respiratory diseases allergic rhinitis (hay fever) and asthma are two of the most common allergic diseases. Both diseases appear to have increased significantly in past two centuries (Seema et al., 1997).

Asthma is a chronic inflammatory disease of the airways which in susceptible individuals causes recurrent episodes of wheezing, breathlessness, chest tightness and cough. Inflammation can increase airway hyper responsiveness which can lead to infections by bacteria including atypical bacteria, fungi and viruses (Brinke et al., 2005).

Recurrent exacerbations are a major cause of morbidity and medical expenditure in patients with asthma. Factors significantly associated with frequent exacerbations include severe nasal sinus disease, gastrointestinal reflux, recurrent respiratory infections, physiological dysfunctioning and obstructive sleep apnoea (David Issacs et al., 2002).

In asthma condition allergic, toxic, fungal, viral and other initiators of inflammation play a major role. There are varieties of triggering factors identified for asthma. Amongst these respiratory tract infections mainly by fungi, viruses and bacteria including atypical bacteria like $M$. pneumoniae and $C$. pneumoniae are important.

Bacterial lipopolysaccharide plays a major role in asthma. Inhaled lipopolysaccharide can exacerbate airway inflammation and airway obstruction in allergic asthmatics. As bacterial infections play a major role in etiopathogenesis of allergic respiratory infections their consequent treatment is required by means of wide spectrum antibiotics as well as prescription of bacterial immunotherapy (Nagayama et al., 1999).

\section{Role of Bacteria in Allergic Respiratory Infections}

In several publications it has been insisted that bacterial infections are important in the etiopathogenesis of bronchial asthma and other allergic respiratory infections. Bacterial antigens potentiate the action of inhalant allergens. It has been already proven that the action of these bacterial antigens increases the number of intraepithelial dendritic cells in the bronchial mucosa after inhalation of bacterial lipopolysaccharide. The structural elements of bacteria and toxins produced by them intensify the release of mediators (Leucotrienes, histamine, IL1, IL 4, IL 6, Il 8 and TNF - alpha) of the inflammatory reactions.

Bacteria commonly found to be associated with respiratory allergies include Moraxella catarrhalis, Streptococcus pneumoniae, $H$. influenzae etc. (Alic Verghese, 2001). Amongst atypical bacteria $M$. pneumoniae and $C$. pneumoniae are associated with bronchial asthma as well as COPD. It is found that bacterial flora of the upper respiratory tract in patients with bronchial asthma is more diverse in comparison with microflora of airways in healthy subjects.

\section{Bacterial Components that Contribute to Allergic reaction}

Of all microbial products, endotoxin has been studied extensively. Along with endotoxins a variety of other microbial 
agents are known to have immune stimulatory properties which includes beta $(1,3)$ - glucans, bacterial DNA and other bacterial components. Beta $(1,3)$ glucans are glucose polymers present in the cell wall of most fungi and yeasts, some bacteria and vegetable materials.

Beta $(1,3)$ glucans have been measured as a marker of mould exposure in field studies. Moulds often grow together with different bacteria and hence levels of beta $(1,3)$ glucan in house dust have been found to be highly correlated with endotoxin levels. Thus endotoxin could be a marker for broader range of microbial exposure.

\section{Bacterial Lipopolysaccharide}

Inhaled LPS can exacerbate airway inflammation and airflow obstruction in allergic asthmatics. Allergic subjects are more sensitive than nonallergic subjects to the bronchoconstrictive properties of inhaled LPS. In addition prior allergen exposure significantly augments the inflammatory response to inhaled LPS.

In healthy nonasthmatic subjects, approximately $10 \%-15 \%$ of subjects are highly sensitive to developing airflow obstruction after inhaling small amounts of LPS, whereas a similar percentage of normal nonasthmatic subjects are highly resistant to developing airflow obstruction after inhalation of high doses of LPS.

\section{Bacterial DNA and Immmunostimulatory DNA CpG sequences}

Bacterial DNA is highly enriched in immunostimulatory DNA containing $\mathrm{CpG}$ motifs that bias the immune response to antigens to a nonallergic Th1 phenotype. This property of bacterial DNA provides a potential mechanism for gastrointestinal bacterial DNA to modulate the developing immune response in infancy. $\mathrm{CpG}$ motifs are a 6-base pair sequence of noncoding DNA that is highly enriched in bacterial DNA.

In mouse models of asthma immunostimulatory DNA sequences inhibit eosinophilic inflammation, Th2 cytokines including IL-5 and airway hyperreactivity to methcholine. The immunostimulatory DNA sequences are effective when administered systemically or mucosally (Henry, 1992).

\section{Mycobacterial Infection and Allergy}

In a study of Japanese schoolchildren, there was a strong inverse association between delayed hypersensitivity to Mycobacterium tuberculosis and atopy.

Positive tuberculin test responses in these children predicted a lower incidence of asthma, lower serum $\operatorname{IgE}$ levels, and cytokine profiles biased towards a Th1 response. Based on this study, it was postulated that exposure to $\mathrm{M}$. tuberculosis may, by modification of immune profiles, inhibit atopic disorder. In mouse models BCG and $M$. vaccae inhibit allergic responses. The potential therapeutic effects of $M$. vaccine in human allergic inflammation are currently being investigated.

Long term use of steroids can give a lot of side effects including suppression of immune system making individual more prone to fungal infections similarly prolonged and irrational use of antibiotic may result in emergence of drug resistance organisms. Unnecessary and random use of medicines without prior diagnosis and also increases total cost of therapy.

\section{Benefit of Antibiotics in Asthma Treatment}

Some macrolides are known to exert immunomodulatory properties that are 
independent of their antibacterial activity. These agents modulate the functions of inflammatory cells, including polymorphonuclear leococytes, lymphocytes and macrophages. Macrolides influence several pathways involved in the inflammatory process, including the migration of neutrophils, the oxidative burst in phagocytes and the production of proinflammatory mediators and cytokines and several of these agents have shown antiinflammatory effects. Macrolides inhibit the synthesis and /or secretion of proinflammatory cytokines (e.g. TNF- $\alpha$, IL8, IL-6, IL-1 $\beta$ ), whereas their effects on anti-inflammatory cytokines (IL-10, IL-4) are more variable.

Objective of the present study was to detect presence of bacterial pathogens in respiratory tract of patients with allergic respiratory conditions such as Asthma \& to check antibiotics effective against these pathogens.

\section{Materials and Methods}

A total of 300 patients were included in the study.

These patients were enrolled for treatment of allergic respiratory disease such as Asthma \& were visiting Medicine Department of T. N. Medical College \& B. Y. L. Nair charitable Hospital, Mumbai Central, Mumbai, Maharashtra, India.

These patients were located in different areas in Mumbai as well as outside Mumbai.

The senior clinician of Medicine department, T. N. Medical College did the selection of subjects on the basis of their clinical and radiological findings.

Patients selected were either hospitalized (Indoor basis) or outdoor patients at B. Y. L. Nair charitable Hospital.

\section{Inclusion Criteria}

1. Patients able to give productive sputum \& clinically suspicious of infective etiology.

2. Patients who are able to produce brief clinical history.

3. Patients in whom antibiotics have not been administered within last 48-72 hours.

\section{Following Exclusion Criteria were used while Selecting Patients for the Study}

1. Patients who are not able to produce adequate sputum or give any other relevant respiratory samples.

2. Patients on prolonged antibiotic treatment.

3. Patients requiring ICU care.

4. Pregnant females.

\section{Collection of Respiratory Specimens}

Sputum samples were collected \& processed. Minimum 3 consecutive samples were studied for confirmation of results. All universal safety precautions were taken while collection, transportation, handling and processing of specimens. All specimens were processed within one hour of collection. Fresh morning sputum specimens were collected with aseptic in a clean, sterile, leak-proof container. Each patient was advised to collect an early morning sputum sample after washing the mouth and gargling with tap water.

All sputum samples were graded by Murray \& Washington grading system \& unsatisfactory samples were discarded. Fresh samples were procured.

\section{Processing of Respiratory Specimens}

All specimens were studied microscopically as well as macroscopically. In macroscopic 
study characters like color, appearance, presence or absence of blood in specimens were noted.

Culture - A loopful of each specimen (Representative portion) was inoculated or streaked on various culture media for isolation of pathogens. All aseptic precautions were taken while processing of samples.

\section{Following media were used}

1. Blood agar

2. Chocolate agar

3. MacConkeys agar

Study of following organisms was excluded

1. Anaerobic organisms

2. Mycobacterium spp.

3. Protozoa

Blood agar and MacConkey agar were incubated aerobically at $37+/-0.5^{\circ} \mathrm{C}$ and growth was observed after 24 hours. If after 24 hours no growth was observed then the plates were incubated further for 24 hours before concluding as No growth. In case growth is observed colony morphology which includes size, shape, consistency, opacity and elevation was recorded.

Blood agar was checked for presence or absence of hemolysis and if present it's type was noted. MacConkeys agar was used to differentiate between lactose fermenters and lactose non-fermenters. Chocolate agar was incubated at $37 \mathrm{C}+/-0.5^{\circ} \mathrm{C}$ in $5-^{\circ} 10 \% \mathrm{CO} 2$ for 48-72 hours. After incubation colony characteristics were noted.

Identification of the isolates obtained Identification of Gram negative bacilli (Lactose fermenting colonies) Identification of Klebsiella pneumoniae

Lactose fermenting (pink) mucoid colonies were obtained on MacConkeys agar which were further identified by performing following biochemical tests

Indole test, Methyl Red test, VP test, Citrate test (IMViC), Motility, Lysine decarboxylase, production, Fermentation of Lactose, Sucrose, Sorbitol, Arabinose, Urease production

Identification of Gram negative bacilli (Lactose non-fermenting colonies)

\section{Identification of Pseudomonas aeruginosa}

Lactose nonfermenting (yellow)colonies were obtained on MacConkeys agar which were further identified by performing following biochemical tests

Oxidase test, Motility, Pigment production, Arginine decarboxylase production, citrate test, Nitrate reduction, Fermentation of Glucose.

Production of bright bluish green diffusible pigment was determined using nutrient agar. $P$. aeruginosa was distinguished from others by it's ability to grow at $4^{0} \mathrm{C}$.

\section{Identification of Gram positive cocci}

\section{Identification of Streptococcus pyogenes}

Small pinpoint beta hemolytic, catalase negative colonies were obtained on Blood agar plate. They were further identified by performing following biochemical tests Bacitracin sensitivity test, Hydrolysis of argfinine, PYR test, Growth inhibition on Bile esculin agar, Trimethoprimsulfamethoxazole resistance.

\section{Identification of Staphylococcus aureus}

Smooth, butyrous, entire edge and about 1-2 $\mathrm{mm}$ in diameter, catalase positive, beta 
hemolytic colonies were obtained on Blood agar plate. They were further identified by performing following biochemical tests

Coagulase test, Voge's-Proskauer test, Nitrate reduction test, Urease test, Anaerobic fermentation of glucose \& mannitol.

\section{Identification of Moraxella catarrhalis}

Non-motile, nonfermentative and oxidase positive. Colonies on chocolate agar gray to white, smooth, opaque. They were further identified by performimg following biochemical tests: Catalase, Gelatin hydrolysis, Nitrate reduction test, Penicillin sensitivity test, Ability to grow on chocolate agar at $22^{\circ} \mathrm{C}$, Ability to grow on nutrient agar at $35^{\circ} \mathrm{C}$.

\section{Antibiotic Susceptibility testing}

Antibiotic susceptibility testing of the organisms of study and control group obtained from clinical specimens was carried out by Kirby-Bauer disk diffusion method according to CLSI guidelines.

A few colonies of the isolate to be tested were inoculated in $5 \mathrm{ml}$ of suitable broth and incubated for 3-4 hours at $37^{\circ} \mathrm{C}$. The turbidity of the broth was matched visually with 0.5 McFarland's standard using sterile saline. A sterile non-toxic cotton swab on a wooden applicator was dipped in to the standardized inoculum. This soaked swab was rotated firmly against the upper inside wall of tube to remove excess fluid. The surface of Mueller Hinton agar plate was uniformly streaked with swab three times by turning the plate at $60^{\circ}$ angle between each streaking.

The plates were allowed to dry at room temperature for 5 mins. Each antibiotic disc was placed aseptically using a pair of sterile forceps on the surface of the culture at an optimum distance on the inoculated plates. The plates were incubated at $37^{\circ} \mathrm{C}$ aerobically.

Plates were incubated at $37^{\circ} \mathrm{C}$ in presence of $5-10 \%$ CO2 for Streptococcus species and Moraxella catarrhalis. Blood agar plates were used to carry out Antibiotic sensitivity pattern for these isolates. For Klebsiella pneumoniae, Pseudomonas aeruginosa, Staphylococcus aureus Mueller Hinton agar plates were used.

The Antibiotic Discs Employed in the Method are as follows

\section{For Klebsiella pneumoniae}

Amikacin (30 mcg), Amox+clav. (30 mcg), Ceftazidime (30 mcg), Cefotaxime (30 mcg), Ciprofloxacin (5 mcg), Gentamicin (10 mcg), Piperacillin (100 mcg), Imipenem (30 mcg)

\section{For Pseudomonas aeruginosa}

Ceftazidime (30 mcg), Cefoperazone (30 $\mathrm{mcg}$ ), Gentamicin (10 mcg), Amikacin (30 $\mathrm{mcg}$ ), Ciprofloxacin (5 mcg), Piperacillin (100 mcg), Aztreonam (30mcg), Imipenem (30 $\mathrm{mcg})$

\section{For Moraxella catarrhalis}

Ampicillin (10 mcg), Ceftazidime (30 mcg), Cefixime (30mcg), Azithromycin (15 mcg), Imipenem (30mcg)., Aztreonam (30 mcg), Tetracycline (30mcg), Cefotaxime (30 mcg)

\section{For Streptococcus pyogenes}

Erythromycin (15 mcg), Vancomycin (30mcg), Ampicillin (10mcg), Clindamycin $(30 \mathrm{mcg})$, Ofloxacin $(5 \mathrm{mcg})$ cefotaxime $(30 \mathrm{mcg})$ ceftazidime $(30 \mathrm{mcg})$ 


\section{For Staphylococcus aureus}

Oxacillin(30 mcg), Vancomycin (30 mcg), Clindamycicn $(30 \mathrm{mcg})$, Erythromycin (15 $\mathrm{mcg})$, Gentamicin $(10 \mathrm{mcg})$, Ciprofloxacin (5mcg), Tetracycline (30 mcg), Amox+clav. (30 mcg),

After overnight incubation at $37^{\circ} \mathrm{C}$, diameter of each zone were measured and recorded in $\mathrm{mm}$. The results were interpreted according to CLSI guidelines, by comparing with the results stated with standard ATCC strains. The pattern obtained was documented as Sensitive, Resistant or Intermediate.

All culture media as well as biochemicals used were procured from HiMedia Laboratories, Mumbai, Maharashtra, India

All media, reagents \& Antibiotic discs were procured from HiMedia Laboratories, Mumbai, Maharashtra, India

Quality control of culture media, Biochemicals \& antibiotic discs was checked by standard methods prescribed.

To confirm their allergic status total serum IgE estimation was also done.

\section{Serological Tests}

3-5 cc of whole blood was collected by venipuncture using a disposable $5.0 \mathrm{ml}$ syringe \& 21 gauge hypodermic needle in a sterile plain test tube taking all aseptic precautions. The sterile plain tube was incubated at $37^{\circ} \mathrm{C}$ in a slanting position for 1-1.5 hours \& later held at $4^{\circ} \mathrm{C}$ for 1 hour. This method facilitates clotting of blood. (This was a routine practice followed at $\mathrm{T}$. N. Medical College \& B. Y. L. Nair charitable Hospital, Mumbai Central, Mumbai, Maharashtra, India where the current study was carried out).
The supernatant serum layer was separated $\&$ centrifuged at $2000 \mathrm{rpm}$ to remove cell debris. (centrifuge REMI, Temperature 28+/$2^{\circ} \mathrm{C}$ ) The clear serum sample was preserved in absence of preservatives in plastic storage vials after labeling properly with patient's registration number $\&$ date at $-20^{\circ} \mathrm{C}$ until utilization. (Maximum capacity of storage vials was $5.0 \mathrm{ml} \&$ were obtained from Himedia Laboratories, Pvt. Ltd., Ghatkopar, Mumbai, Maharashtra, India)

The stored serum was used for detecting Total IgE by solid phase ELISA

(Details are mentioned below)

*Total IgE estimation was done by solid phase Enzyme Linked Immunoassay by IBL ELISA. (Quantitative)

This is IBL manufactured kit. Kit contained 6 standard samples which had $\mathrm{IgE}$ concentrations from $0 \mathrm{IU} / \mathrm{ml}$ to $1000 \mathrm{IU} / \mathrm{ml}$.

On a semi-logarithmic graph paper the concentrations of the standards (abscissa logarithmic) were plotted against their corresponding optical density (ordinate linear) The concentration of the samples can be read directly from this standard curve by using their average optical density. Any sample reading greater than the highest standard should be diluted appropriately. The result has to be multiplied with corresponding dilution factor.

As all the above ELISA used were quantitative titers were determined $\&$ then interpretation was done.

\section{Results and Discussion}

In recent decades it is seen that bacterial infection factor has been overlooked in the causal treatment of bronchial asthma and allergies. But literature evidence suggests that bacterial infections play a major role in 
the etiopathogenesis of allergic diseases. Therefore along with treatment for allergic manifestations it is necessary to give treatment for these bacterial infections with wide spectrum antibiotics. This will result in cure in asthmatic patients without maintaining them on inhalers and unnecessary corticosteroid therapy.

In our study $20 \%$ allergic cases showed presence of bacterial etiology in their respiratory tract, as determined by culture oftheir sputum sample. M. catarrhalis was found to be most prominent isolate obtained in $31.66 \%$ cases, followed by $S$. aureus obtained in $28.33 \%$ cases. Other isolates obtained included $K$. pneumoniae \& $P$. aeruginosa both in $16.66 \%$ cases each. $S$. pyogenes was obtained in $6.66 \%$ cases. (Table 1) Similar findings are reported by Nagayama and Tsubaki T et al.

Nagayama and Tsubaki et al cultured sputum specimens quantitatively from asthmatic children aged 0-14 years in order to determine the relationship between asthmatic status and bacterial species present in the respiratory tract.

In children with acute asthma attack, $H$. influenzae, Streptococcus pneumoniae, $M$. catarrhalis were distributed evenly in the samples. In patients with prolonged asthma attack pathogenic bacteria were present in $34.7 \%$ of patients. In these patients $H$. influenzae was predominant. In patients with pneumonia without asthma attack $40.9 \%$ of pathogenic bacteria were obtained. This study showed that there is a significant relationship between the presence of bacteria in sputum and clinical symptoms such as fever and pneumonia episodes during acute asthma attacks.

Antibiotic sensitivity testing was performed by standard Kirby -Bauer disc diffusion method as prescribed by CLSI. All $S$. pyogenes isolates (total 4) \& All $M$. catarrhalis isolates (total 19) were sensitive to all the antibiotics tested

All S. aureus isolates (Total 17) were sensitive to oxacillin, augmentin, Clindamycin, gentamicin, ciprofloxacin, Tetracycline \& vancomycin. 70\% S. aureus isolates were sensitive to Erythromycin. Erythromycin resistance was observed in $30 \%$ isolates. As reported by Shrestha and Singh et al an in vitro study has documented cloxacillin as the most effective antibiotic for $S$. aureus \& ciprofloxacin \& erythromycin as the least effective antibiotics.

Levels of erythromycin resistance have reached $20-40 \%$ in some parts of the world. Steady increase of MIC from $11.5 \mathrm{mcg} / \mathrm{ml}$ in $2.7 \%$ isolates in 1980 to $>100 \mathrm{mcg} / \mathrm{ml}$ in 5 . $1 \%$ isolates in 1985 have been reported $n$ India.

At present around $80-90 \%$ of strains of $M$. catarrhalis are shown to acquire resistance to ampicillin in recent years. 24\% strains from Southern India were found to have reduced susceptibility to co-trimoxazole.

In present study all $M$. catarrhalis isolates (Total 19) obtained were sensitive to all antibiotics tested.

All isolates of $K$. pneumoniae obtained (Total 10) in the present study were sensitive to cefotaxime, ceftazidime, Amikacin, Ciprofloxacin \& Imipenem. Augmentin resistance was observed in $50 \%$ isolates, Gentamicin \& Piperacillin resistance was observed in $20 \%$ isolates for each antibiotic.

In case of $P$. aeruginosa isolates obtained (Total 10) were sensitive to cefotaxime, ceftazidime, Amikacin, Aztreonam 
Imipenem \& piperacillin. Gentamicin \& ciprofloxacin resistance was observed in $20 \%$ isolates for each antibiotic.

Shrestha \& Singh et al reported amikacin as the most effective drug for Gram negative bacteria \& cephalexin as the least effective drug.( Shrestha et al., 2005)

Patients gave history of single or multiple criteria like previous history of allergic disease, known allergy, history of pulmonary disease, history of addiction, sensitivity to dust, fumes and cold products.

Patients enrolled in the study often complained about chest pain, fever, breathlessness and exacerbations and sputum purulence. One or more of the above symptoms might be due to bacterial infections. Total immunoglobulin levels were also found to be elevated above normal levels in few cases. There is also a possibility that these bacteria may themselves act as allergens and aggreviate the already existing allergic conditions. Based on the diagnosis of allergic manifestation corticosteroid, inhaler therapy is subscribed taking into consideration the allergic condition. But at the same time presence of bacterial agents in respiratory tract of allergic patients should be also considered and antibiotics specific for that particular pathogen. The effective combination of steroid therapy and focused antibiotic therapy will result in complete cure of allergic condition.

In the present study total serum IgE levels were estimated by ELISA as total IgE levels provide the evidence in support of atopy. All patients showed IgE levels above $1000 \mathrm{IU} / \mathrm{ml}$, which correlated with respiratory symptoms, history \& atopy.

Chowdhary and Vinaykumar et al reported elevated IgE levels in $90 \%$ allergic rhinitis cases. In their study of allergic rhinitis associated with bronchial asthma cases, IgE values were more than $1000 \mathrm{IU} / \mathrm{ml}$. They also proved that $90 \%$ patients with allergic rhinitis with peripheral eosinophil counts in normal ranges. When rhinitis was associated with bronchial asthma, the eosinophil values showed an increase above the normal.

In conclusion, respiratory infections are important causes of diseases and disability worldwide. They are the most common cause of death in underdeveloped countries, especially in children. Allergic conditions affect $20 \%$ of the world's population, amongst which respiratory allergies are important ones.

Study of respiratory infections in allergic conditions generally includes study of the risk factors, associated symptoms and clinical history. Microbial etiology is often overlooked.

Previous studies has proved that microorganisms appear to be involved in some of the cases the etiology of some of the cases of allergic manifestations. Hence, there is a need to study the role of microorganisms in allergic conditions, which will help in identifying the exact cause of the exacerbations and symptoms of allergic conditions. Focused treatment considering these infectious agents is important in minimizing the severity of allergic conditions. Infectious etiology can itself act as an allergen aggravating the allergic as well as COPD conditions. 
Table.1 Showing number of bacterial isolates in sputum specimens collected from Asthma patients

\begin{tabular}{|c|c|c|c|c|c|c|}
\hline \multirow{2}{*}{$\begin{array}{l}\text { Total } \\
\text { number of } \\
\text { samples } \\
\text { processed }\end{array}$} & \multirow{2}{*}{$\begin{array}{l}\text { Total number of } \\
\text { samples showing } \\
\text { positive bacterial } \\
\text { culture findings }\end{array}$} & \multicolumn{5}{|c|}{ Bacterial isolates } \\
\hline & & M. catarrhalis & S. pyogenes & S. aureus & K. pneumoniae & P. aeruginosa \\
\hline 300 & $\begin{array}{c}60 \\
(20 \%)\end{array}$ & $\begin{array}{c}19 \\
(31.66 \%)\end{array}$ & $\begin{array}{c}04 \\
(6.66 \%)\end{array}$ & $\begin{array}{c}17 \\
(28.33 \%)\end{array}$ & $\begin{array}{c}10 \\
(16.66 \%)\end{array}$ & $\begin{array}{c}10 \\
(16.66 \%)\end{array}$ \\
\hline
\end{tabular}

Along with steroid therapy antibiotic targeting the infectious agents can be given which will result in minimizing the cost of treatment and side effects associated with irrational and random use of antibiotics and steroids.

Hence there is an urgent need to focus on the microbial etiology associated with infections in allergic conditions.

The main objective of this prospective longitudinal study was to determine the etiology and clinical history of patients suffering from infections in allergic conditions

\section{Acknowledgements}

Our special thanks to Dr. S. N. Oak, Former Dean, T.N. Medical College \& B. Y. L. Nair Charitable Hospital, Mumbai Central, Mumbai, Maharashtra, India \& Dr. S. T. Nabar, Former Head, Medicine Department, T.N. Medical College \& B. Y. L. Nair Charitable Hospital, Mumbai Central, Mumbai.

\section{References}

Alic Verghese. 2001. Immune Profile in Respiratory Viral Infections in Asthmatics. Indian J. Allergy Immunol., 15(2): 87-92 .

Bauer, A.W., Kirby, W.M.M., Sheries, J.C., Turuck, M. 1966. Antibiotic susceptibility testing by standard single disk method. American $J$.
Pathol., 5: 433-496.

CLSI. 2014. guidelines Performance Standards for Antimicrobial Susceptibility Testing $24^{\text {th }}$ informational supplement M00 s24.

David Greenwood, Richard, C. B. 1992. Black Medical Microbiology $14^{\text {th }}$ edition, ELBS Churchill Livingstone.

David Issacs, Preeti Joshi. 2002. Respiratory infections and Asthma MJA, 177(6 suppl): S50-S51.

David, H. Broide. The Role of Bacterial infections in Allergy : A clinical Paradox Review from $57^{\text {th }}$ Annual Meeting of the American Academy of Allergy, Asthma and Immunology, March 16-21, 2001, New Orleans, Louisiana.

Ekiel, A., Friedek, D., Szulakowski, Romanik, M. 2005. Incidence of selected bacterial pathogens of the respiratory tract in patients with bronchial asthma Wiad Lek, 55(12): 11-8.

Elmer, W., Konemann Stephen, D., Allen, William, M., Janda. 1992. In Colour Atlas and Textbook of diagnostic Microbiology, $14^{\text {th }}$ edition, J. B. Lippincott Company

Frankel, S., S. Reitman, S, Sonnenwirth, A.C. 1970. Gradwohl's Clinical Laboratory methods and diagnosis: $7^{\text {th }}$ edition: vol. I and vol. II: C. V. Mosby Company.

Gehring, U., J. Heinrich, G. Hoek, M. Giovannangelo. 2007. Bacteria and mould components in house dust and children's allergic sensitization. 
Eur. Respir. J., 29: 1144-1153.

Gupta, P.R., D.K. Mangal. 2006. Prevalence of risk factors for bronchial asthma in adults in Jaipur district of Rajasthan. Lung India, 23: 53-58.

Henry, D. 1992. Isenberg Clinical Microbiology Procedures Handbook, Vol I, 1992, American Soc. Microbiol.

Holt, S.J.G., N.R. Krieg, P.H.A. Sneath et al. 1994. Bergeys Manual of Determinative Bacteriology. $9^{\text {th }}$ edition; William and Wilkins, Baltimore.

Jindal, S.K. 2006. Respiratory Disease Epidemiology In India. Lung India, 23: 93-94.

Nagayama Y., Tsubaki, T. 1999. Role of bacterial infection in the exacerbation of caute or prolonged asthma attack in children Allergol. Int., vol. 48, no.02, pp 137-144(8).

Oehling, A.K. 1999. Bacterial infection as an important triggering factor in bronchial asthma. J. Investg. Allergol Clin. Immunol., 9(1): 6 -13.

Reba Kanungo, A. Kumar, S. Bhattacharya. 2000. Antimicrobial Resistance among common bacterial respiratory pathogens Indian $J$. Med. Microbiol., 18(2): 55-61.

Reeves, S., D.S., Philips, I., Williams, J. D., Wise, R. 1978. Laboratory methods in antimicrobial chemotherapy, Churchill Livingstone.
Revised National Tuberculosis Control Programme (RNTCP). 1999. Manual for Laboratory Technicians Central TB division, Directorate General of Heath services, Ministry of health and Family Welfare, Nirmal Bhavan, New Delhi.

Schaechter, M., G. Medeff, D. Schlessinger. 1989. Mechanisms of Microbial Disease International edition, Williams and Wilkins.

Sebastian, L., Johnston, Richard, J. 2005. Martin Chlamydophila pneumoniae and Mycoplasma pneumoniae : A Role in Asthma Pathogenesis ? Am. J. Respir. Crit. Care Med., Vol. 172, pp 1078 -1089.

Seema, M.T., P.R. Bindhu. 1997. Serum Immunoglobulins in Asthma Indian J. Med. Microbiol., 15(2): 77-78.

Shrestha, U., Singh, A., B.M. Pokhrel. Cross-sectional study of respiratory pathogens and their antibiotic susceptibility patern in Tribhuvan University Teaching Hospital. $J$. Institute of Med., 28: 2: 5-9.

Tarfe, K.S., Deshpande, S.D. 2015. A typical bacteria \& Candida: Threats for allergic respiratory diseases. Life Sci. Int. Res. J., vol 2 spl Issue, ISSN 2347-8691.

Ten Brinke, A., P.J. Sterk, A.A.M. 2005. Risk factors of frequent exacerbations in difficult to treat asthma. Eur. Resp. J., 26: 812 818

\section{How to cite this article:}

Kishori S. Tarfe and Sunita D. Deshpande. 2016. Bacterial Etiology: Significant In Allergic Respiratory Diseases. Int.J.Curr.Microbiol.App.Sci. 5(6): 577-587. doi: http://dx.doi.org/10.20546/ijcmas.2016.506.062 